Account

\title{
Large-scale Molecular Dynamics Simulations for Analyses of Transport Phenomena in Polymer Electrolyte Fuel Cell
}

\author{
Takashi TOKUMASU*a, Akinori FUKUSHIMA ${ }^{\text {a }}$, Takuya MABUCHI ${ }^{\mathrm{b}}$ and Yuta SUGAYA ${ }^{\mathrm{b}}$
}

\author{
anstitute of Fluid Science, Tohoku University \\ ${ }^{\mathrm{b}}$ Graduate School of Engineering, Tohoku University \\ *e-mail: tokumasu@ifs.tohoku.ac.jp
}

(Received: August 25, 2012; Accepted for publication: November 22, 2012; Advance publication: March 12, 2013)

\begin{abstract}
Computer simulation is a very powerful tool to analyze transport phenomena in the membrane electrode assembly (MEA) of polymer electrolyte fuel cells (PEFCs). In particular, there are many nanoscale structures in this flow field, and therefore the phenomena should be analyzed from the microscopic point of view rather than computational fluid dynamics. In this paper, we report large-scale molecular dynamics (MD) simulations to analyze these flows. In particular, dissociation phenomena of a hydrogen molecule on a Pt catalyst, transport phenomena of proton and water in a polymer electrolyte membrane (PEM), oxygen permeability of ionomers in a catalyst layer (CL), and transport phenomena of a water droplet in a nanopore were simulated, and their characteristics are discussed. In the analysis of the dissociation phenomena of the hydrogen molecule, it was found that the trend of dissociation probability as a function of impinging energy considering the motion of the molecule differs from that without considering the motion of the molecule. In the analysis of proton transfer in a PEM, the diffusion coefficients obtained by this simulation were consistent with the experimental data. In the analysis of oxygen permeability of ionomers, the dependence of water content on the permeability was estimated and the difference between ionomer on catalyst layer and that in bulk state was clarified. In the analysis of transport phenomena of a water droplet in a nanopore, we compared the results of our simulation with the macroscopic governing equation.
\end{abstract}

Keywords: Molecular dynamics, Polymer electrolyte fuel cell, Transport phenomena, Water content

\section{Introduction}

In recent years, problems involving carbon dioxide have greatly increased, and new power sources which use fossil fuels efficiently are becoming important. Under these circumstances, fuel cells, especially the polymer electrolyte fuel cell (PEFC), have rapidly improved and are being commercialized. To achieve a compact and low-cost PEFC system, the development of a high-performance membrane electrode assembly (MEA) under high temperature, low humidity and low pressure is very important. Fundamental knowledge of transport phenomena in a MEA is required to design high-performance MEAs under these conditions. Computer simulations, as well as many experimental schemes, are very powerful tools to analyze the transport phenomena, and many conventional computer simulations based on computational fluid dynamics with constitutive equa- tions based on electrochemistry or experimental results have been performed [1]. However, the flow fields in MEAs, such as the gas diffusion layer (GDL), microporous layer (MPL), catalyst layer (CL) and polymer electrolyte membrane (PEM), are in the order of nanometers to micrometers, and therefore the macroscopic simulation cannot reproduce the transport phenomena in PEFC even qualitatively.

In this paper, we analyze such nanoscale transport phenomena in a PEFC by large-scale molecular dynamics simulations and discuss the results, particularly focusing on the difference from conventional macroscopic theory. In this paper, we introduce four topics that strongly affect the efficiency of PEFCs: dissociation phenomena of hydrogen molecules on a Pt catalyst, proton and water transport in a polymer electrolyte membrane, oxygen permeation through the ionomer in a cathode CL, and the transport mechanism of water droplets in a nanopore. 
Through these simulations, we analyze the nanoscale characteristics of these flow phenomena and the relation between them and the nanoscale structure of these flow fields. Moreover, the differences between the macroscopic and microscopic points of view and the different mechanisms are discussed.

\section{Dissociation phenomena of hydrogen molecules on a Pt catalyst}

In this study, the dissociation phenomena of a hydrogen molecule on a Pt catalyst were simulated by molecular dynamics simulations and the dependence of the motion of the molecule on the dissociation probability was analyzed. A schematic diagram of this simulation is shown in Figure 1. In real system, ionomer covers Pt surfaces and affects the impinging energy of $\mathrm{H}_{2}$ molecules. Therefore, this simulation differs much on the realistic dissociation phenomena. However, the potential used in this simulation needs much calculation load and it is impossible to analyze dissociation phenomena with complex ionomer molecules. For this reason, we consider the dissociation phenomena without ionomer and focus on only the difference of the trend of dissociation phenomena when the phenomena was considered with or without the motion of molecules.

In this simulation, the interaction potential considering the electron density should be used because dissociation of a hydrogen molecule occurs by transferring an electron to the nonbonding orbital near the Pt surface. The embedded atom method (EAM) [2] was used in this study. In EAM, the form for the potential energy, $E_{\text {pot}}$, of the system that consists of $N \mathrm{Pt}$ atoms and a $\mathrm{H}_{2}$ molecule is given by

$$
\begin{aligned}
E_{\mathrm{pot}} & =\sum_{i=1}^{N} F_{\mathrm{Pt}}\left(\rho_{i}\right)+\sum_{k=1}^{2} F_{\mathrm{H}}\left(\rho_{k}\right)+\frac{1}{2} \sum_{i}^{N} \sum_{j(\neq i)}^{N} \phi_{\mathrm{Pt}-\mathrm{Pt}}\left(R_{i j}\right) \\
& +\phi_{\mathrm{H}-\mathrm{H}}\left(R_{12}\right)+\sum_{i=1}^{N} \sum_{k=1}^{2} \phi_{\mathrm{Pt}-\mathrm{H}}\left(R_{i k}\right),
\end{aligned}
$$

where subscripts $i$ and $j$ denote $\mathrm{Pt}$ atoms, $k$ denotes a $\mathrm{H}$ atom and $R$ is the distance between the atoms. The function, $F(\rho)$, is the energy to embed an atom into the background electron

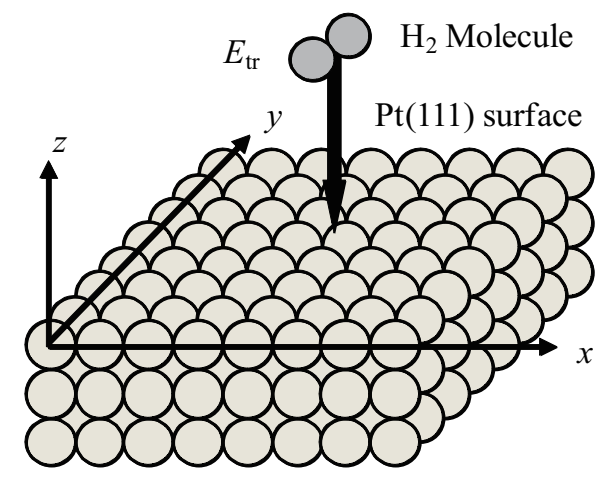

Figure 1. Schematic diagram of the simulation system for dissociation of a hydrogen molecule on a Pt catalyst.

density, and expresses the interaction between an atom and the electrons of the system. The functions, $F_{\mathrm{Pt}}$ and $F_{\mathrm{H}}$, are the energies necessary to embed a $\mathrm{Pt}$ atom and a $\mathrm{H}$ atom, respectively. The function $\varphi(R)$ is the pair interaction potential between atoms separated by a distance $R$, and $\varphi_{\mathrm{Pt}-\mathrm{Pt}}, \varphi_{\mathrm{Pt}-\mathrm{H}}$ and $\varphi_{\mathrm{H}-\mathrm{H}}$ are the pair interaction potentials between two Pt atoms, a Pt atom and a $\mathrm{H}$ atom and two $\mathrm{H}$ atoms, respectively. The functions $\rho_{i}$ and $\rho_{k}$ are the electron densities at the position of $\mathrm{Pt}$ atom $i$ and $\mathrm{H}$ atom $k$, respectively, contributed by the surrounding atoms. In EAM, the electron densities, $\rho_{i}$ and $\rho_{k}$, are assumed to be superpositions of the electron density contributed by each surrounding atom. The functions used in Eq. (1) were determined so that the energy characteristics of the Pt crystal or a $\mathrm{H}_{2}$ molecule, the electron density around a $\mathrm{H}$ atom or on the $\mathrm{Pt}$ (111) surface obtained by the EAM potential were consistent with those obtained by density functional theory (DFT) results [3]. To check the validity of the potential, the dissociation barrier at each site and for a specific orientation was calculated using the EAM potential and the results were compared with those obtained by DFT [4]. The results are shown in Table 1. As shown in this table, the dissociation barriers are consistent with those from DFT and the validity of the potential is verified.

In Figure 1, the Pt surface was constructed of $300 \mathrm{Pt}$ atoms aligned in three layers in the $z$-direction, each of which consists

Table 1. Dissociation barriers at each site and for a specific orientation.

\begin{tabular}{lccccccc}
\hline \multirow{2}{*}{ site } & $\boldsymbol{\Phi}$ & \multicolumn{3}{c}{ EAM } & \multicolumn{2}{c}{ DFT [4] } \\
\cline { 2 - 7 } & {$[\mathbf{d e g}]$} & $\mathbf{R}[\AA]$ & $\mathbf{Z}[\AA]$ & $\mathbf{E b a r}[\mathbf{e V}]$ & $\mathbf{R}[\AA]$ & $\mathbf{Z}[\AA]$ & $\mathbf{E b a r}[\mathbf{e V}]$ \\
\hline brg & 0 & 0.86 & 2.03 & 0.23 & 0.82 & 1.87 & 0.27 \\
& 90 & 0.92 & 1.67 & 0.41 & 0.82 & 1.66 & 0.40 \\
top & 0 & 0.80 & 2.27 & 0.09 & 0.77 & 2.25 & 0.06 \\
& 90 & 0.80 & 2.27 & 0.09 & 0.77 & 2.28 & 0.06 \\
fcc & 0 & 0.89 & 1.82 & 0.33 & 0.84 & 1.70 & 0.42 \\
\hline
\end{tabular}


of $10 \mathrm{Pt}$ atoms $(27.72 \AA)$ in the $x$-direction and $10 \mathrm{Pt}$ atoms (24.00 $\AA$ ) in the $y$-direction. Periodic boundary conditions were imposed in the $x$ - and $y$-directions. The initial positions of the $\mathrm{Pt}$ atoms were the same as the configuration of a Pt bulk system. The lattice constant of Pt was set at $3.92 \AA$. The temperature of the system was controlled at $T=350 \mathrm{~K}$. After the surface reached an equilibrium state, $\mathrm{a}_{2}$ molecule impinged at the specific sites (top, brg and fcc) of the Pt surface from a height of $5 \AA$. The impinging energy, $E_{\mathrm{tr}}$, was given to the $\mathrm{H}_{2}$ molecule. The rotational energy, $E_{\text {rot }}$, was set to 0 . The direction of the velocity of the molecule was given as normal to the Pt surface, and the orientation of the molecule was given randomly. The velocity Verlet algorithm [5] was used. In this simulation, the $\mathrm{H}_{2}$ molecules collide with the Pt surface and some $\mathrm{H}_{2}$ molecules dissociate and some do not. In this paper, the $\mathrm{H}_{2}$ molecules whose internuclear distance between $\mathrm{H}$ atoms becomes more than $3.5 \AA$ are defined as having dissociated [3]. These simulations were performed 640 times, fixing the impinging energy and the site on the Pt (111) surface, and changing the orientation of the $\mathrm{H}_{2}$ molecule, and the dissociation probability was obtained as a function of impinging energy at top, brg and fcc sites. The initial configuration of the Pt (111) surface was changed in every simulation.

The dynamic dissociation probabilities as a function of impinging energy are shown in the upper part of Figure 2. The static dissociation probability obtained using the EAM potential and transition state theory is shown in the lower part of Figure 2 [3]. As shown in this Figure, at the brg and fcc sites, the dynamic dissociation probability increases with increasing impinging energy of the $\mathrm{H}_{2}$ molecule, and becomes nearly constant at higher impinging energy, which is similar to the static dissociation probability. At the top site, however, the dynamic dissociation probability decreases with increasing impinging energy. This tendency is very different from the static dissociation probability shown in the lower part. Moreover, the lower part shows that the dissociation probability when the impinging energy is near 0 is about $25 \%$, which means that $75 \%$ of the impinging molecules cannot pass over the dissociation barrier. However, the upper part shows that $90 \%$ of the impinging molecules dissociate when the impinging energy is near 0 . This contradiction shows the dynamic effects on the dissociation probability. The behavior of the impinging $\mathrm{H}_{2}$ molecules clearly shows that $\mathrm{H}_{2}$ molecules with very low impinging energy can reach the chemisorption layer by changing their orientation via interaction with the Pt (111) surface [3]. These impinging molecules have no dissociation barrier, even if they have an initial orientation at which the dissociation barrier is large. The change in orientation of an impinging molecule via interac-
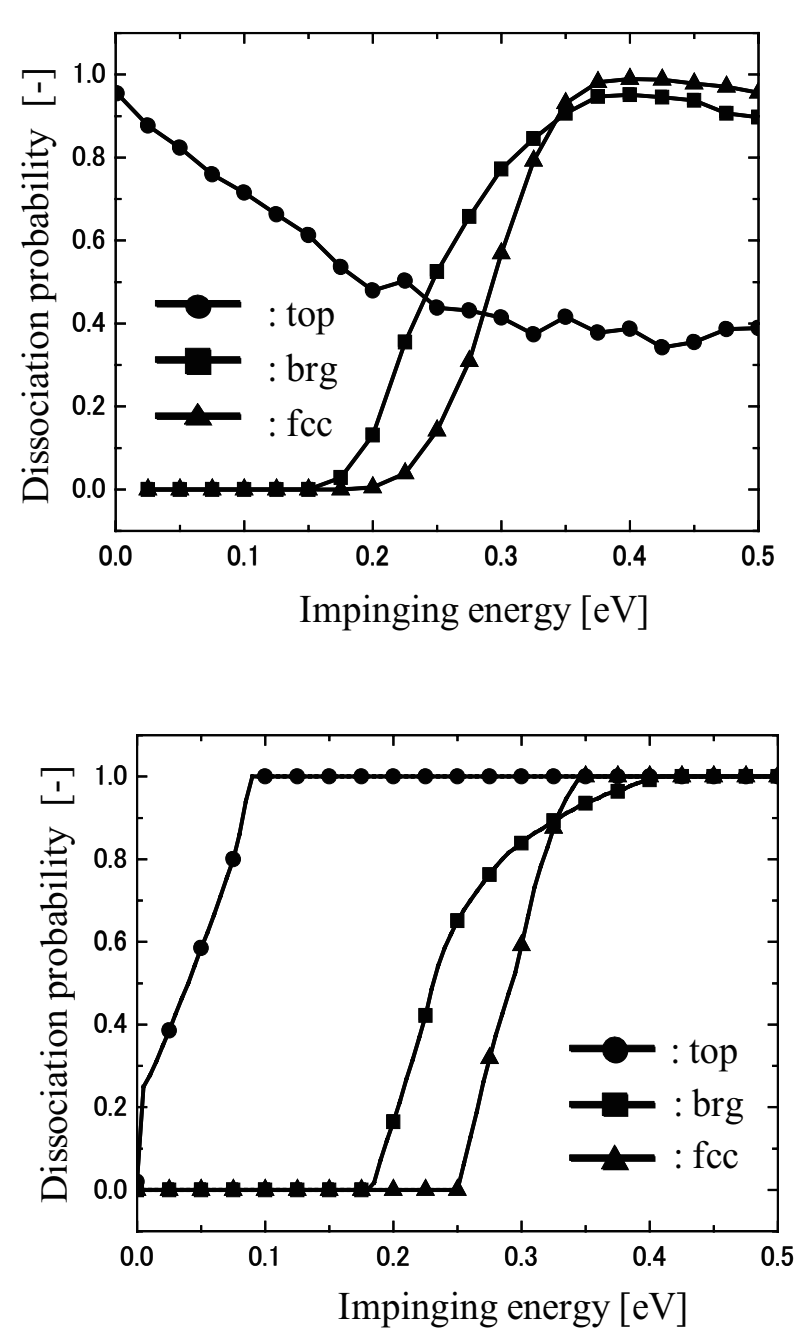

Figure 2. Dynamic dissociation probability (upper) and static dissociation probability (lower).

tion with the surface to reduce the dissociation barrier is called the "steering effect" [6]. The steering effect is also observed at the brg and fcc sites. The impinging molecules with very low impinging energy, however, cannot pass over the dissociation barrier regardless of orientation at brg or fcc sites, because the minimum dissociation barrier is more than $0.1 \mathrm{eV}$ at the brg and fcc sites. For this reason, the steering effect is unimportant for dissociation phenomena at the brg or fcc sites. It was concluded that the dynamic effect has to be considered in estimating the dissociation probability for surfaces at which the dissociation barrier is relatively low. 


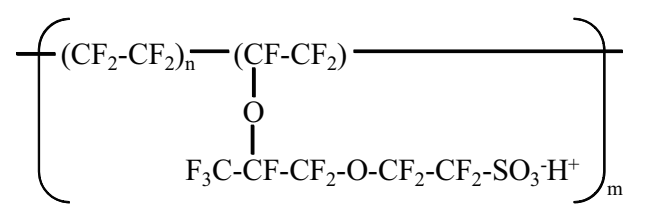

Figure 3. Chemical structure of a PFSA molecule. In this study, $n=7$ and $m=13$.

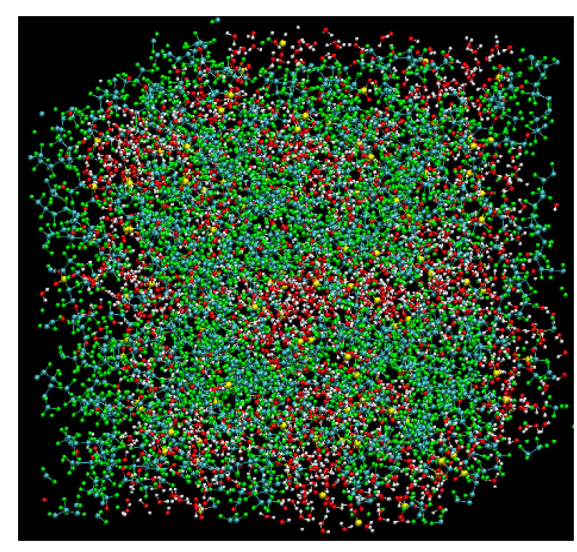

Figure 4. Schematic diagram of the simulation system for proton and water transport phenomena.

\section{Proton and water transport in PEM}

In this study, the transport mechanism of protons and water in the nanostructure of hydrated perfluorosulfonic acid (PFSA) membrane was analyzed by classical molecular dynamics simulation. There are two mechanisms for proton transfer, the Vehicle and Grotthuss mechanisms. We only analyze the transport phenomena using the Vehicle mechanism in the present study. It was assumed that all sulfonate groups $\left(\mathrm{SO}_{3} \mathrm{H}\right)$ were completely ionized to $\mathrm{H}^{+}$and $\mathrm{SO}_{3}^{-}$. A PFSA molecule was made by linking the polar monomeric unit $(\mathrm{P})$ to the end of the nonpolar monomeric unit $\mathrm{N}_{7}$, performing the procedure 13 times to obtain $\left(\mathrm{N}_{7} \mathrm{P}\right)_{13}$, in the notation of Jang et al. [7], in the case of which $n=7$ and $m=13$ as shown in Figure 3. $\mathrm{A} \mathrm{CF}_{3}$ groups were used to terminate the main chain. The potential model for the PFSA molecule was based on the Dreiding force field $[7,8]$ and the torsion parameters of the potential were modified to fit the experimental properties of PFSA membrane. The flexible three-center (F3C) model [9] for water molecules and classical hydronium model [7] for hydronium ions were employed.

The initial configurations were generated by randomly placing 25 PFSA chains in a box, $x \times y \times z=200 \times 200 \times 200 \AA^{3}$, with periodic boundary conditions in all directions. To ensure
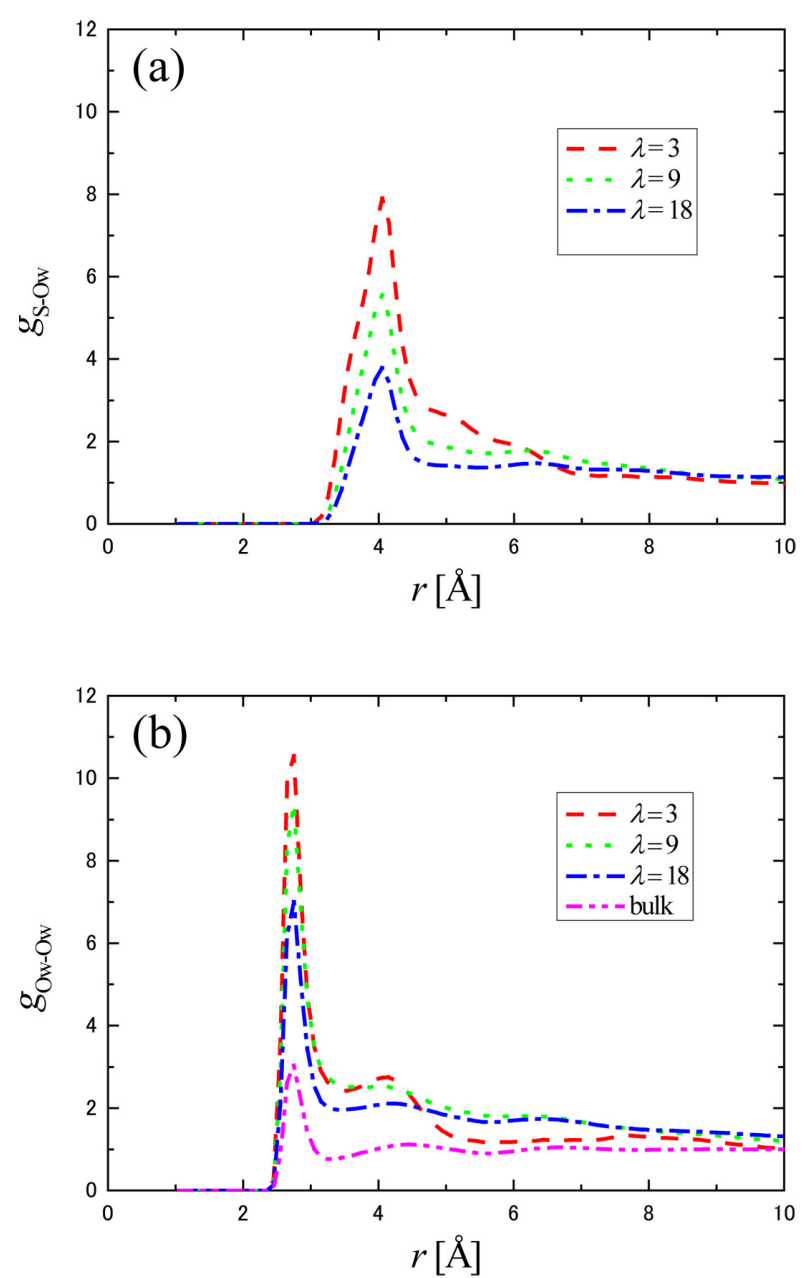

Figure 5. Radial distribution function (RDF) in membrane as a function of water content, $\lambda$. Figure (a) shows RDF between $\mathrm{S}$ atom of sulfo group and $\mathrm{O}$ atom of $\mathrm{H}_{2} \mathrm{O}$ mnolecule. Figure (b) shows RDF between $\mathrm{O}$ atoms of $\mathrm{H}_{2} \mathrm{O}$ molecule.

charge neutrality, we added a total of $25 \times 13=325$ hydronium ions and considered water contents of $\lambda=1,3,6,12$ and 18 , where the parameter $\lambda$ indicates the ratio of the number of water molecules to the number of $\mathrm{SO}_{3}^{-}\left(N_{\mathrm{H} 2 \mathrm{O}, \mathrm{H} 3 \mathrm{O}_{+}} / N_{\mathrm{SO}_{3}-}\right)$. After all of the molecules were added, the annealing procedure was carried out to eliminate the initial configuration and orientation of the molecules and to establish an equilibrium state. The density of the simulation system after annealing was calculated and the results were compared with experimental data. It was confirmed that the density variation rate as a result of the annealing procedure was less than $0.1 \%$ and the system can express the real conditions of PFSA membranes at each water content. The temperature was held constant by the Nosé-Hoover thermostat [10], and the pressure was controlled by the Andersen method [11]. 

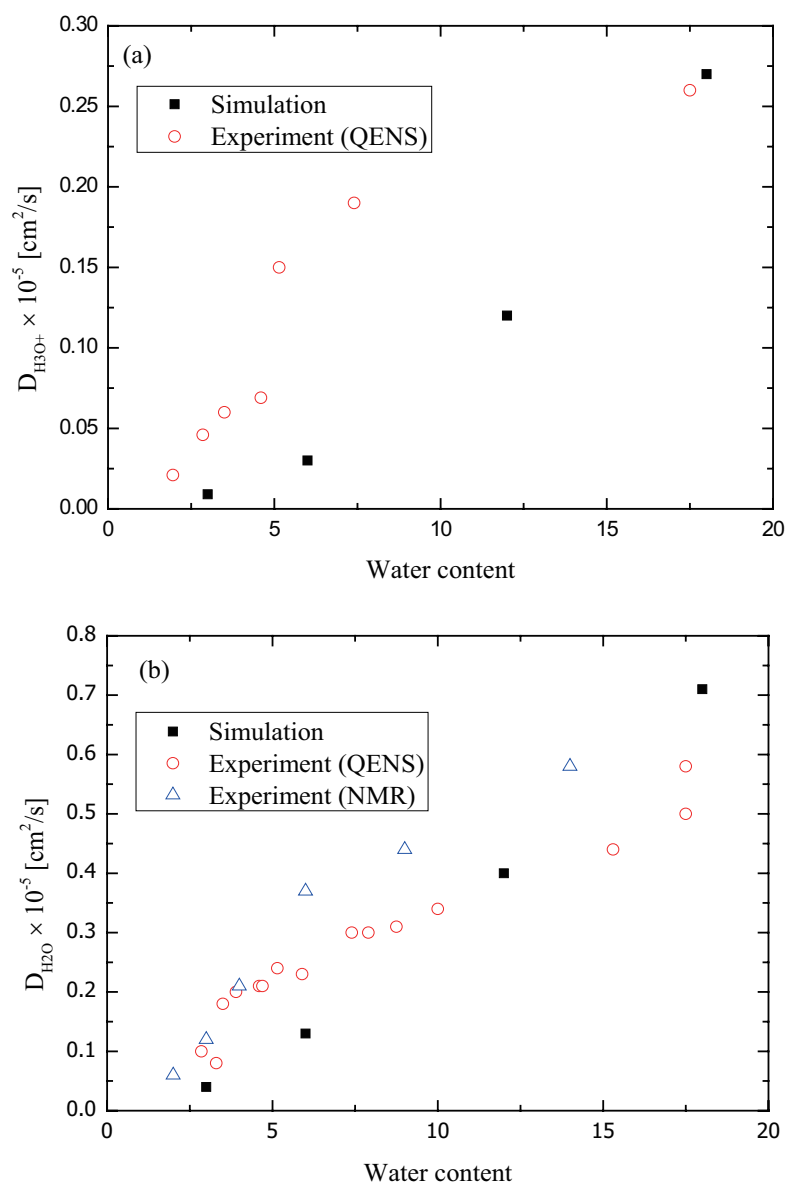

Figure 6. Diffusion coefficient of (a) hydronium ions and (b) water molecules as a function of water content, $\lambda$.

The multiple-time method, r-RESPA [12], was employed for the time integration technique, such as intramolecules for $1 \mathrm{fs}$, intermolecules for $2 \mathrm{fs}$ and the long-distance term in the PME method for 4 fs. The final simulation system is shown in Figure 4.

First the structure of water molecules is analyzed by radius distribution function (RDF) in a membrane. RDF is defined as the ratio of number density at a certain distance from a molecule to the average number density [5]. The RDFs at $\lambda=3,9,18$ are shown in Figure 5. Figure 5 (a) shows the RDF between $\mathrm{S}$ atom of sulfo group and $\mathrm{O}$ atom of $\mathrm{H}_{2} \mathrm{O}$ molecule and Figure 5 (b) shows the RDF between $\mathrm{O}$ atoms of $\mathrm{H}_{2} \mathrm{O}$ molecule. As shown in Figure 5 (a), the first peak of RDF decrease with the increase in the water content, that describes water in membrane is highly structured when the water content is lower. Moreover, as shown in Figure 5 (b), RDF depends on the water content and the structure of water molecules is different from that of bulk water.
Using this simulation technique, the mobility of the hydronium ions and water molecules was analyzed by calculating the mean square displacements (MSDs) of the hydronium ions and the water molecules. The diffusion coefficients, $D$, for the oxygen of hydronium ions $\left(\mathrm{O}_{\mathrm{h}}\right)$ and water molecules $\left(\mathrm{O}_{\mathrm{w}}\right)$, were obtained using the MSDs to satisfy the Einstein-Smoluchowski function obtained by

$$
D=\lim _{t \rightarrow \infty} \frac{1}{6 t}\left\langle|r(t)-r(0)|^{2}\right\rangle,
$$

where $t$ is the diffusion time and $r(t)$ is the position vectors of $\mathrm{O}_{\mathrm{h}}$ and $\mathrm{O}_{\mathrm{w}}$. The diffusion coefficients were obtained from the linear regime of the MSD curves. The results are shown in Figure 6. As shown in the upper part, the diffusion coefficient of the hydronium ion obtained by experimental results is much higher than that obtained by our simulation. This is because our simulation method cannot treat the Grotthuss mechanism and therefore the diffusivity of the proton is estimated to be lower than the real value. The diffusion coefficients of water, however, are in good agreement with experimental data $[13,14]$ and these results testify to the validity of our calculation models, which use only the Vehicle mechanism.

\section{Oxygen permeation through the ion- omer in the cathode $\mathbf{C L}$}

In this study, the effect of water content on oxygen permeability of the ionomer was analyzed using molecular dynamics. An ionomer was made from the PFSA molecule, including hydronium ion and water molecules. Pt-Pt interactions were represented by a spring-mass model considering nearest-neighbor atoms; the spring constant and equilibrium distance were set at $k=46.8 \mathrm{~N} / \mathrm{m}$ and $r_{e}=0.392 \mathrm{~nm}$, respectively. The interaction potential of PFSA, water and hydronium ion is the same as for the PEM simulation. A two-center Lennard-Jones (LJ) model [15] was used for the oxygen molecule. The interaction between Pt and water was represented by the Spohr and Heinzinger potential (SH potential) [16] and that between Pt and hydronium ion was represented by the potential in which the number of hydrogen atoms changes from two to three. The other potentials were connected by the LJ potential, calculated using Lorentz-Berthelot mixing rules; however, the $\mathrm{Pt}-\mathrm{O}_{2}$ interaction was not considered so that oxygen molecules that reach the platinum surface go through the surface without any barrier, assuming that all oxygen molecules react on the platinum surface and disappeared immediately. An oxygen molecule that goes through the platinum surface was put in the upper region of the surface so that a constant mass flux was generated in the 


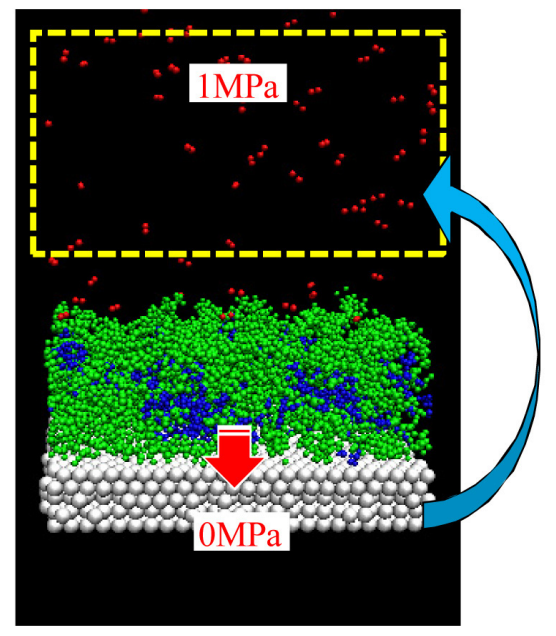

Figure 7. Schematic diagram of the simulation system for oxygen permeability of ionomer in the cathode CL.

simulation system.

The simulation domain was set at $66.5 \times 57.6 \times 150.0 \AA^{3}$. As the initial state, five Pt layers were placed in the $z$-direction; the 1 st to 3 rd layers were harmonic layers, the 4 th layer was a phantom layer to maintain the temperature and the 5th layer was fixed. There were 1024 atoms in each layer. A chain of PFSAs was constructed: five pairs of one polar chain and six nonpolar chains $\left(\mathrm{N}_{6} \mathrm{P}_{1}\right)_{5}$, which is the case of $n=6$ and $m=5$ in Figure 3. Fifteen PFSAs were placed in this system. $\mathrm{CF}_{3}$ groups were used to terminate a main chain. In addition, the number of water molecules and hydronium ions was determined according to the water content of $\lambda=3,7$ and 11 . The initial velocity of each molecule was obtained according to the Boltzmann distribution at the temperature of $350 \mathrm{~K}$. The time-integration scheme is the same as for the PEM simulation. First, the ionomer without the Pt surface was annealed for $800 \mathrm{ps}$, so that the temperature of the system first increased to $700 \mathrm{~K}$ and next decreases by the

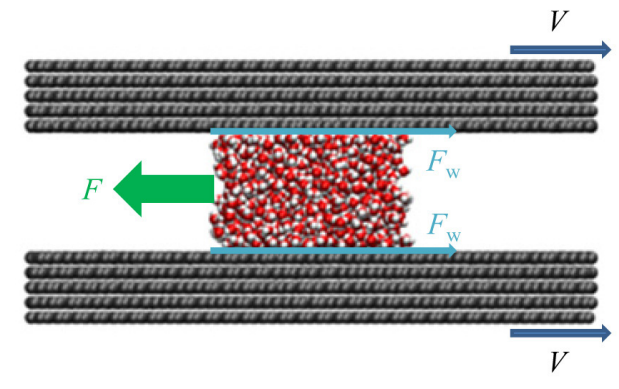

Figure 8. Schematic diagram of the simulation system for the transport phenomena of a water droplet in a nanopore.

velocity-scaling method. The volume of the system decreases so that the water molecules in the ionomer do not leave. Over the next $800 \mathrm{ps}$, ionomer was adsorbed by the Pt surface. After the system reached the equilibrium state, oxygen molecules were added to the simulation system so that the pressure gradient between the upper and lower regions of the Pt surface was $1 \mathrm{MPa}$. A schematic diagram of the simulation system is shown in Figure 7.

The number of oxygen molecules that permeate through the ionomer and the permeability coefficient as a function of the water content are shown in Table 2. As shown in Table 2, oxygen permeability of the ionomer on catalyst surface decreases with the increase in water content, a trend that is opposite to that of the experimental data using bulk PFSA membrane. To analyze the inconsistency between the simulation and experimental results, the oxygen permeability of ionomer in its bulk state was simulated. In the bulk region, the permeability coefficient is expressed by the product of the diffusion coefficient and the solubility coefficient. In this study, the diffusion coefficient was obtained from the Einstein-Smoluchowski function by Eq. (2) and the solubility coefficient was obtained from the chemical potential [17] by

Table 2. Number of oxygen molecules and the permeability coefficient, $P$, as a function of water content.

\begin{tabular}{lccc}
\hline Water content, $\lambda$ & $\mathbf{3}$ & $\mathbf{7}$ & $\mathbf{1 1}$ \\
\hline Number of permeated oxygen molecules & 36 & 4 & 3 \\
Permeability coefficient $\left(\times 10^{-9}\right)\left[\mathrm{cm}^{3}(\mathrm{STP}) / \mathrm{cm} / \mathrm{s} / \mathrm{Pa}\right]$ & 5.0409 & 0.6145 & 0.4945 \\
\hline
\end{tabular}

Table 3. Permeability coefficient of PFSA membrane and its components as a function of water content.

\begin{tabular}{lccc}
\hline Water content, $\boldsymbol{\lambda}$ & $\mathbf{3}$ & $\mathbf{7}$ & $\mathbf{1 1}$ \\
\hline Permeability coefficient $\left(\times 10^{-11}\right)\left[\mathrm{cm}^{3}(\mathrm{STP}) / \mathrm{cm} / \mathrm{s} / \mathrm{Pa}\right]$ & 1.292 & 1.472 & 1.665 \\
Solubility coefficient $\left(\times 10^{-6}\right)\left[\mathrm{cm}^{3}(\mathrm{STP}) / \mathrm{cm}^{3} \mathrm{~Pa}\right]$ & 1.162 & 0.854 & 0.708 \\
Diffusion coefficient $\left(\times 10^{-5}\right)\left[\mathrm{cm}^{2} / \mathrm{s}\right]$ & 1.112 & 1.725 & 2.351 \\
\hline
\end{tabular}




$$
\mu=-k_{B} T \ln s_{0}=-k_{B} T \ln \left\langle\exp \left(-\frac{\Delta U}{k_{B} T}\right)\right\rangle,
$$

where $\Delta U$ is the energy difference when an oxygen molecule is put in the PFSA membrane, $k_{\mathrm{B}}$ is the Boltzmann constant, $T$ is the temperature and $s_{0}$ is the entropy. The results are shown in Table 3. As shown in Table 3, the permeability coefficient of ionomer in its bulk state increases with an increase in the water content, and therefore it is confirmed that the trend of the simulation result is consistent with the experimental data. Moreover, these results showed that the reason why the oxygen permeability increases with an increase in the water content is that the diffusivity of oxygen increases, while the solubility of oxygen decreases as a function of the water content. The results indicate that the characteristics of oxygen permeability are different between the ionomer on catalyst layer and that in bulk state, although the material is the same.

\section{Transport mechanism of a water droplet in a nanopore}

In this study, the transport properties of a water droplet in the nanohydrophobic pore were analyzed focusing on the difference between its dynamics and the macroscopic dynamics. Figure 8 shows a schematic diagram of the simulation system for the transport properties of a water droplet in a nanohydrophobic pore. Graphite consisting of five layers was assumed for the hydrophobic walls. Each wall consists of 8000 atoms. Some different-sized micropores were modeled by the parameter $W$, which is the distance between the two walls. In this study, four micropore models were used, where $W$ was set at 10, 20, 30 and $40 \AA$. Periodic boundary conditions were imposed on these models. In our calculations, a $39.4 \AA \times 106.5 \AA \times 3 W$ super cell was taken for all calculations. If $3 W$ was shorter than $100.0 \AA$, the cell length in the $z$-direction was set at $100.0 \AA$ so that the unit cell has a sufficiently large vacuum region to make the interaction with molecules in the next cell along the $z$-direction negligible. The number of water molecules was defined by the parameter $D$. Thus, the number of water molecules $\left(N_{w}\right)$ was obtained by

$$
N_{w}=D L_{x} L_{y}\left(W-W_{0}\right) / M_{\text {wat }},
$$

where $M_{\text {wat }}$ is the mass of a water molecule, and $L_{x}$ and $L_{y}$ are the cell lengths along the $x$ - and $y$-directions, respectively. $W_{0}$ denotes the volume where water molecules cannot exist because of the repulsion force from the walls and was set at $5.0 \AA$. In this study, four $D$ values, $200,300,400$ and $500 \mathrm{~kg} / \mathrm{m}^{3}$, were

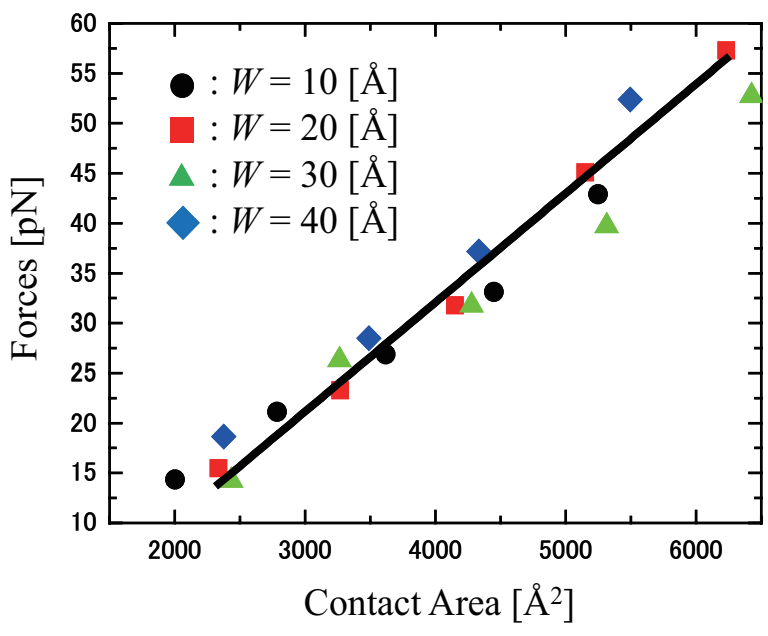

Figure 9. Forces acting on a water droplet from the walls.

employed. To achieve the condition that the water droplet has a constant velocity, the outermost layers were moved along the $y$-direction with constant velocity, and an extra force that is equal in magnitude and opposite in direction was added to the water droplet to prevent it from accelerating. The force was equally divided over all water molecules. To keep the system at a constant temperature, the temperature of the fourth layer was kept constant in all time steps using the velocity-scaling method. The SPC/E model [18] was used as the water molecule model. The LJ potential was chosen as the interaction between the oxygen atom in a water molecule and the atoms of the walls [19]. The two-body harmonic oscillator potential was used for the interactions between atoms in the walls.

First, the force acting on the water droplet was analyzed. In the macroscopic point of view, the force was expressed by

$$
F=2 F_{w}=2 \sigma L_{x}\left(\cos \theta_{b}-\cos \theta_{f}\right)+2 \alpha S V,
$$

where $\sigma$ denotes the surface tension, $\theta_{f}$ and $\theta_{b}$ denote the forward and backward contact angles, respectively, $L_{x}$ denotes the length of the simulation system in the $x$-direction, $S$ denotes the contact area and $\alpha$ denotes the friction coefficient. In Eq. (5), the first term denotes the contribution of surface tension and the second term denotes that of the friction force. In the molecular dynamics simulation, $F$ is obtained by the total force acting on the water molecules from the wall molecules. The results are shown in Figure 9. These total forces are independent of the width of the micropore and linearly depend on the contact area. The slope of the line is evaluated as $9.29 \times 10^{5} \mathrm{~N} / \mathrm{m}^{2}$, and the intercept of this line is $6.24 \times 10^{-1} \mathrm{pN}$. It means that the disappearance of the bulk region in the center of the micropore 
model for $W=10 \AA$ does not affect the magnitude of the total forces. Next, we compare these results with Eq. (5). Considering that $L_{x}$ is constant, the first term in Eq. (5), which means the force generated by the surface tension, is the intercept of the interpolated line. In this case, the contribution of surface tension is very small. The reason is considered that the contact angle of the water droplet against the graphite surface is very small. In future work, we will perform the same simulation by changing the contact angle, and the effect of wettability of a nano pore on the force acting on the droplet will be analyzed.

\section{Summary}

We performed large-scale molecular dynamics simulations to analyze nanoscale transport phenomena in PEFC using a supercomputer. In particular, we analyzed the dissociation phenomena of a hydrogen molecule on a Pt catalyst, proton and water transport in a PEM, oxygen permeation through an ionomer in the cathode $\mathrm{CL}$ and the transport mechanism of a water droplet in a nanopore, which affects the efficiency of PEFCs. From the results of this simulation, we can obtain information about the nanoscale flow phenomena of proton, oxygen and water, which is difficult to obtain by experiment or macroscopic simulations. This knowledge helps us to design a new concept of MEA for higher efficiency and lower cost of PEFCs in the future.

I am grateful to my staff for providing me with the main simulation results described in this paper. A part of this work was supported by the New Energy and Industrial Technology Development Organization (NEDO) of Japan, and the simulations were mainly performed using the supercomputer at the Institute of Fluid Science (IFS) of Tohoku University.

\section{References}

[1] Y. Wang, K. S. Chen, J. Mishler, S. C. Cho, X. C. Adroher, Appl. Energ., 88, 981 (2011). [CrossRef]

[2] M. I. Baskes, Phys. Rev. B, 46, 2727 (1992). [CrossRef]

[3] T. Tokumasu, D. Ito, J. Appl. Phys., 109, 063509 (2011). [CrossRef]

[4] R. A. Olsen, G. J. Kroes, E. J. Baerends, J. Chem. Phys., 111, 11155 (1999). [CrossRef]

[5] M. P. Allen, D. J. Tildesley, Computer Simulation of Liquids, Clarendon Press, Oxford, U.K., (1986).

[6] G. R. Darling, S. Holloway, J. Chem. Phys., 101, 3268 (1994). [CrossRef]

[7] S. S. Jang, V. Molinero, T. Cagin, W. A. Goddard, J. Phys. Chem. B, 108, 3149 (2004). [CrossRef]

[8] T. Li, A. Wlaschin, P. B. Balbuena, Ind. Eng. Chem. Res., 40, 4789 (2001). [CrossRef]

[9] M. Levitt, M. Hirshberg, R. Sharon, K. E. Laidig, V. Daggett, J. Phys. Chem. B, 101, 5051 (1997). [CrossRef]

[10] W. G. Hoover, Phys. Rev. A, 34, 2499 (1986). [Medline] [CrossRef]

[11] H. C. Andersen, J. Chem. Phys., 72, 2384 (1980). [CrossRef]

[12] M. Tuckerman, B. J. Berne, G. J. Martyna, J. Chem. Phys., 97, 1990 (1992). [CrossRef]

[13] J. C. Perrin, S. Lyonnard, F. Volino, J. Phys. Chem. C, 111, 3393 (2007). [CrossRef]

[14] T. A. Zawodzinski, M. Neeman, L. O. Sillerud, S. Gottesfeld, J. Phys. Chem., 95, 6040 (1991). [CrossRef]

[15] M. Bohn, R. Lustig, J. Fischer, Fluid Phase Equilib., 25, 251 (1986). [CrossRef]

[16] E. Spohr, K. Heinzinger, Ber. Bunsenges. Phys. Chem, 92, 1358 (1988).

[17] M. Fukuda, J. Phys. Chem, 112, 478 (2000). [CrossRef]

[18] H. J. C. Berendsen, J. R. Grigera, T. P. Straatsma, J. Phys. Chem., 91, 6269 (1987). [CrossRef]

[19] T. Werder, J. H. Walther, R. L. Jaffe, T. Halicioglu, P. Koumoutsakos, J. Phys. Chem. B, 107, 1345 (2003). [CrossRef] 